\title{
Wnt signaling regulates smooth muscle precursor development in the mouse lung via a tenascin C/PDGFR pathway
}

\author{
Ethan David Cohen,1,2,3 Kaori Ihida-Stansbury,4,5 Min Min Lu,1,3 Reynold A. Panettieri, ${ }^{1}$ \\ Peter Lloyd Jones, ${ }^{4,5,6}$ and Edward E. Morrisey1,2,3,7
}

\begin{abstract}
${ }^{1}$ Department of Medicine, ${ }^{2}$ Department of Cell and Developmental Biology, ${ }^{3}$ Cardiovascular Institute,
${ }^{4}$ Department of Pathology and Laboratory Medicine, 5 Institute for Medicine and Engineering, ${ }^{6}$ Penn-CMREF Center for Pulmonary Hypertension Research, and ${ }^{7}$ Institute of Regenerative Medicine, University of Pennsylvania, Philadelphia, Pennsylvania, USA.
\end{abstract}

\begin{abstract}
Paracrine signaling from lung epithelium to the surrounding mesenchyme is important for lung SMC development and function and is a contributing factor in an array of pulmonary diseases such as bronchopulmonary dysplasia, pulmonary hypertension, and asthma. Wnt7b, which is exclusively expressed in the lung epithelium, is important for lung vascular smooth muscle integrity, but the underlying mechanism by which Wnt signaling regulates lung SMC development is unclear. In this report, we have demonstrated that Wnt7b regulates a program of mesenchymal differentiation in the mouse lung that is essential for SMC development. Genetic loss-of-function studies showed that $\mathrm{Wnt} 7 \mathrm{~b}$ and $\beta$-catenin were required for expression of Pdgfr $\alpha$ and Pdgfr $\beta$ and proliferation in pulmonary SMC precursors. In contrast, gain-of-function studies showed that activation of Wnt signaling increased the expression of both Pdgfr $\alpha$ and Pdgfr $\beta$ as well as the proliferation of SMC precursors. We further showed that the effect on Pdgfr expression was, in part, mediated by direct transcriptional regulation of the ECM protein tenascin C (Tnc), which was necessary and sufficient for Pdgfr $\alpha / \beta$ expression in lung explants. Moreover, this pathway was highly upregulated in a mouse model of asthma and in lung tissue from patients with pulmonary hypertension. Together, these data define a Wnt/Tnc/Pdgfr signaling axis that is critical for smooth muscle development and disease progression in the lung.
\end{abstract}

\section{Introduction}

The mammalian lung is a highly complex organ that develops from the anterior foregut through interactions between the anterior foregut endoderm and the surrounding mesenchyme. Early in development epithelial-mesenchymal interactions pattern the lung endoderm and mesenchymal components leading to distinct cells types along the proximal-distal axis of the lung. These patterning events are essential for proper development of both the airway epithelial cells required for gas exchange and surfactant expression and development of the pulmonary vasculature and airway smooth muscle. Some of the key pathways that regulate these epithelial-mesenchymal interactions include Vegf, Bmp, Shh, and Wnt, but the mechanisms by which they regulate lung mesenchymal development, in particular smooth muscle development, are poorly understood (reviewed in ref. 1). Defects in these developmental processes lead to several important lung diseases, including pulmonary hypertension and asthma. For example, mutations in the bone morphogenetic protein receptor 2 gene are a leading cause of familial pulmonary hypertension, underscoring the link between important developmental pathways and adult lung disease $(2,3)$.

Wnt signaling is a key regulator of multiple aspects of tissue development. The canonical Wnt pathway involves the stabilization and nuclear translocation of $\beta$-catenin through the binding of Wnt ligands to the frizzled/LRP coreceptor complex. $\beta$-Catenin acts as a powerful trans-activator of LEF/TCF transcription factors, regulating important downstream target genes that promote

Conflict of interest: The authors have declared that no conflict of interest exists. Citation for this article: J. Clin. Invest. 119:2538-2549 (2009). doi:10.1172/JCI38079. cell proliferation, differentiation, and tissue development. Despite a growing list of general target genes, less is known about the tissue-specific targets for this pathway. In the lung, Wnt signaling has been shown to regulate airway epithelial differentiation, proliferation, and vascular smooth muscle development (4-8). In particular, loss of Wnt7b leads to decreased mesenchymal differentiation and proliferation and later in development decreased VSMC integrity and pulmonary hemorrhage (8). VSMCs in Wnt7 blacZ mutants exhibits either a hypertrophic and apoptotic appearance or significant thinning, which suggests that defective VSMC development leads to hypertrophy, cell death, and subsequent thinning of the vessel wall and hemorrhage (8). Since Wnt7b is expressed exclusively in the developing airway epithelium, these data demonstrate important epithelial-mesenchymal signaling by Wnt7b that regulates lung VSMC development and function.

Given the importance of Wnt signaling in lung epithelialmesenchymal interactions and lung development in general, we sought to define the molecular program acting downstream of Wnt7b and $\beta$-catenin signaling in the development of smooth muscle precursors in the mesenchyme. Using in vivo gain- and loss-of-function analyses combined with differential gene expression assays, we define a molecular program regulated by Wnt7b and $\beta$-catenin through tenascin C (Tnc), Pdgfr $\alpha$, and Pdgfr $\beta$, which are important for smooth muscle precursor development. Importantly, we find that this Wnt/Tnc/Pdgfr pathway is upregulated in mouse and human models of asthma and pulmonary hypertension. These data provide important insight into the role for Wnt signaling in lung SMC development, providing evidence that Wnt signaling regulates early smooth muscle precursor differentiation in the lung as well as influences SMC development in the adult lung. 

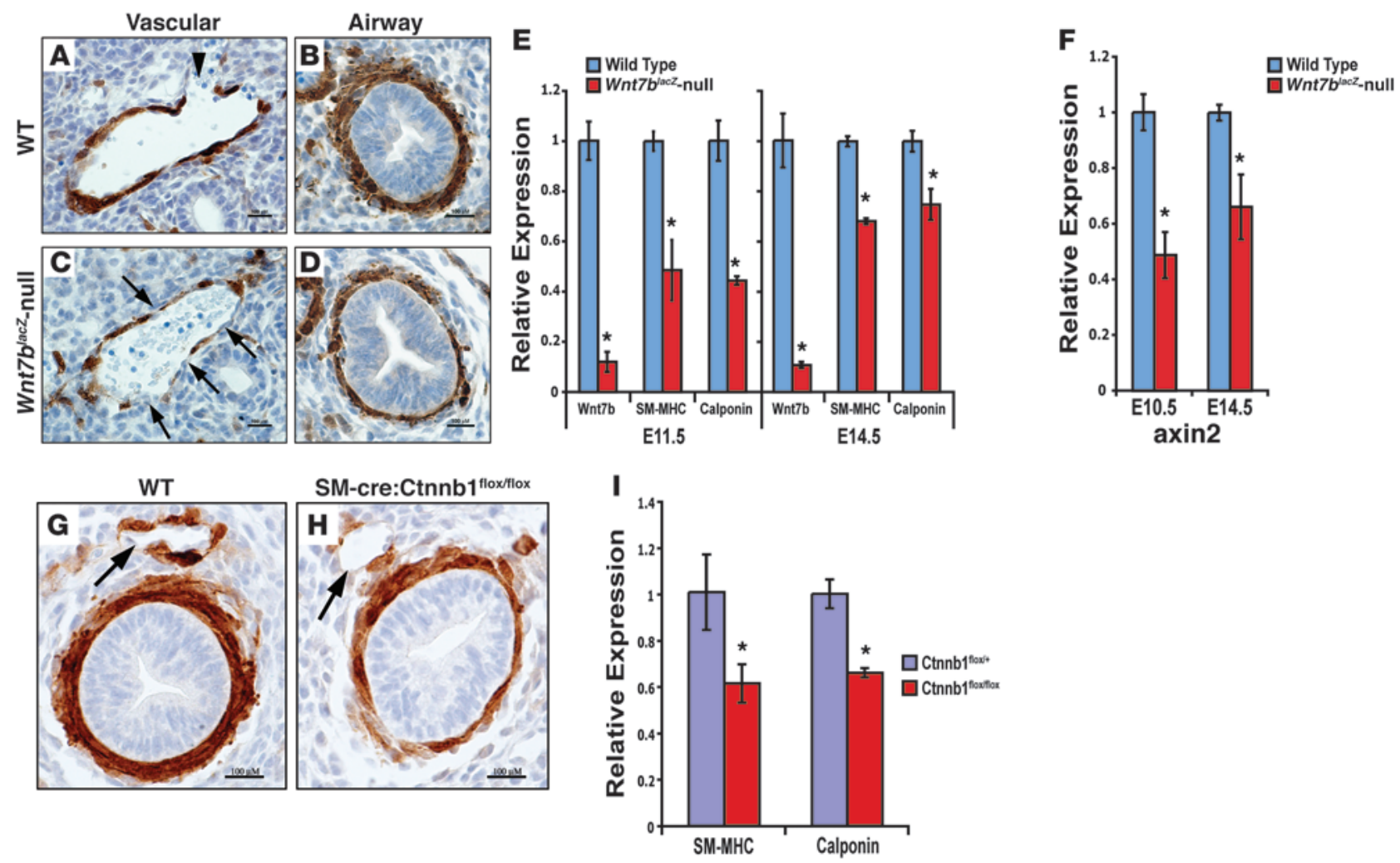

\section{Figure 1}

Loss of Wnt7b expression leads to early defects in SMC development in the lung. (A-D) SM22 $\alpha$ immunostaining showed vascular (A and C) and airway (B and $\mathbf{D})$ smooth muscle development in wild-type (A and $\mathbf{B})$ and $W n t 7 b^{\text {lacz-null }}(\mathbf{C}$ and $\mathbf{D})$ mutants. Arrowhead in $\mathbf{A}$ indicates a smaller branching vessel in wild-type embryos, and arrows in $\mathbf{C}$ point to gaps in SM22 $\alpha$ expression in surrounding larger blood vessels of Wnt7 $\mathbf{b}^{\prime a c z}$-null mutants. (E) Loss of Wnt7b expression in Wnt7 $b^{1 a c Z}$-null mutants led to decreased expression of other SMC markers, including SM-MHC and calponin, as shown by Q-PCR. (F) Wnt7 blacZ-null mutants exhibited decreased axin2 expression. (G and $\mathbf{H})$ SM22 $\alpha$-cre:Ctnnb1 ${ }^{\text {flox/flox }}$ mutants at E12.5 exhibited decreased SM22 $\alpha$ expression surrounding both airways and blood vessels (arrows) compared with wild-type littermates. (I) SM22 $\alpha$-cre:Ctnnb1 floxflox mutants had decreased SM-MHC and calponin expression as shown by Q-PCR. ${ }^{*} P<0.01$. Scale bars: $100 \mu \mathrm{m}$.

\section{Results}

Wnt $7 b$ and $\beta$-catenin are required for vascular and airway smooth muscle development in the developing lung. To address the underlying molecular mechanism of Wnt7b function in SMC development in the lung, immunohistochemistry for the smooth muscle-specific protein SM22 $\alpha$ was performed on sections through the lungs of control and $W n t 7 b^{l a c Z}$ mutant embryos at E14.5. In wild-type lungs, a contiguous layer of SM $22 \alpha$-positive cells was observed surrounding large blood vessels, with gaps in SM22 $\alpha$ expression restricted to the openings of smaller vessels while a thick layer of SMCs encircled the developing proximal airways (Figure 1, A and B). In contrast, the layer of SM $22 \alpha$-positive cells surrounding similarly sized vessels and airways in $W_{n t 7 b^{l a c Z}}$ mutant lungs was consistently thinner than those found in control lungs with large gaps in SM22 $\alpha$ expression, and the layer of SMCs surrounding the proximal airways was also thinner (Figure 1, C and D). These data suggest that early defects in SMC development leads to the later loss of these cells in $W n t 7 b^{\text {lac } Z}$ mutants (8).

To quantify the effect of loss of Wnt7b expression on pulmonary SMC development, expression of the $\mathrm{Ca}^{2+}$ binding protein calponin-h1 and smooth muscle myosin heavy chain (SM-MHC) was examined by quantitative real-time PCR (Q-PCR). At E11.5 and E14.5, calponin-h1 and SM-MHC were significantly reduced in the lung buds of Wnt7b mutants (Figure 1E). The decreasing severity of this loss at E14.5 may be due to compensatory upregulation of the related ligand Wnt7a after loss of Wnt7b expression (Supplemental Figure 1; supplemental material available online with this article; doi:10.1172/JCI38079DS1) (8, 9). We also examined the expression of axin2, a well-characterized target of Wnt/ $\beta$-catenin signaling that provides an accurate readout of signaling activity, in wild-type and $W n t 7 b^{l a c Z}$ mutant lungs (10). Axin2 expression was reduced at both E10.5 and E14.5 in Wnt7blac mutants, indicating that loss of Wnt7b signaling leads to decreased $\beta$-catenin-dependent transcription (Figure $1 \mathrm{~F}$ ).

To determine whether $\beta$-catenin (Ctnnb1) was required for lung smooth muscle development, we examined expression of SM $22 \alpha$ in SM22 $\alpha$-cre:Ctnnb1flox/flox mutants. Of note, the SM $22 \alpha$-cre line expresses the cre recombinase in smooth muscle precursors as well as definitive SMCs, and the Ctnnb $1^{\text {flox/flox }}$ allele contains loxP sites flanking exons 2-6 and upon cre recombination results in a null allele $(11,12)$. As predicted, control embryos at E12.5 had a thick ring of SM22 $\alpha$ expression surrounding the large airways and developing blood vessels (Figure $1 \mathrm{G}$ ). In contrast, the smooth muscle layer was thinner and SM22 $\alpha$ staining less intense surrounding the large airways and developing blood vessels of SM22 $\alpha$-cre:Ctnnb1 flox/flox mutants (Figure $1 \mathrm{H}$ ). Consistent with the apparent reduction in SM $22 \alpha$ staining seen in the SM22 $\alpha$-cre:Ctnnb1flox/flox mutant lungs, expression of the smooth muscle marker genes calponin-h1 and 

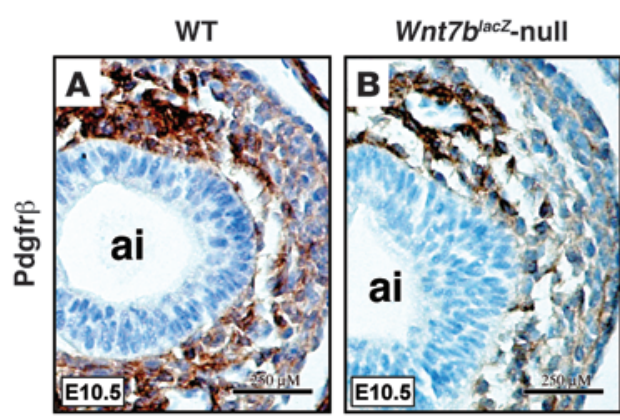

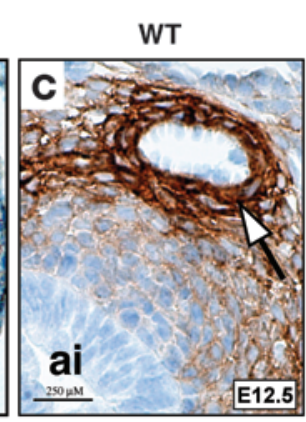

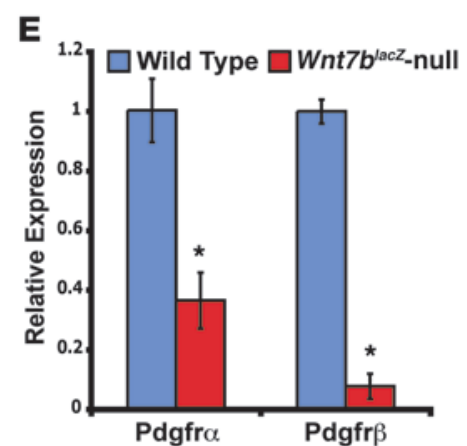

Pd
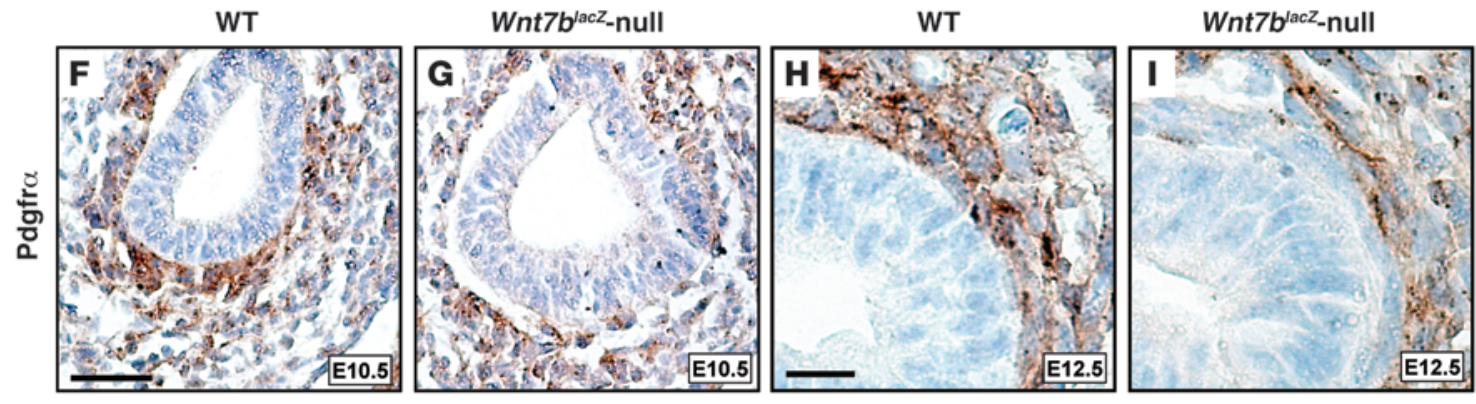
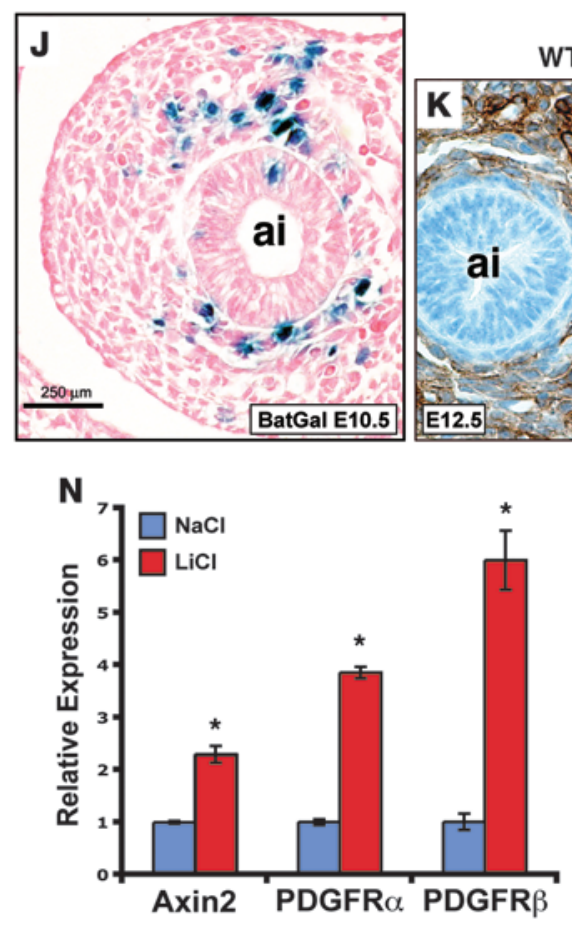

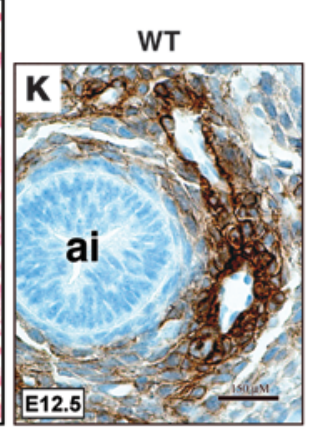

SM-cre:Ctnnb1 $1^{\text {floxtlox }}$
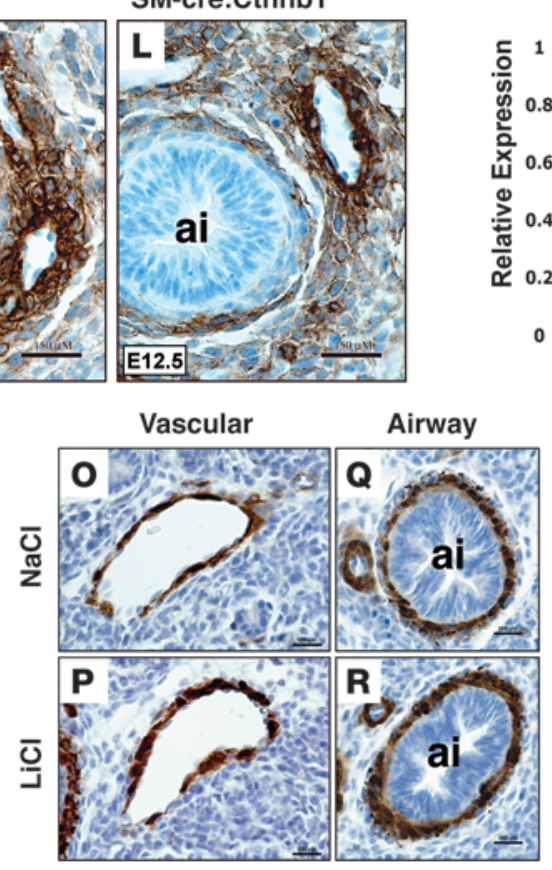

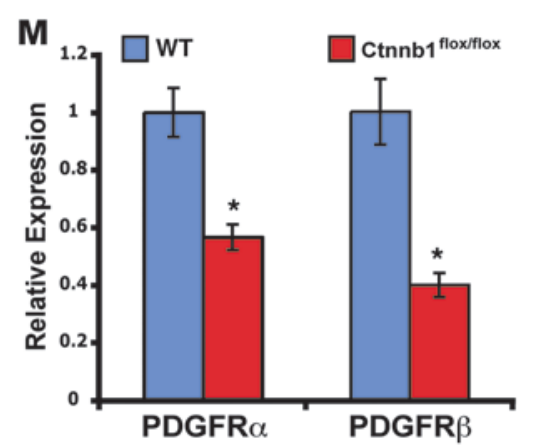

$\mathbf{S}$

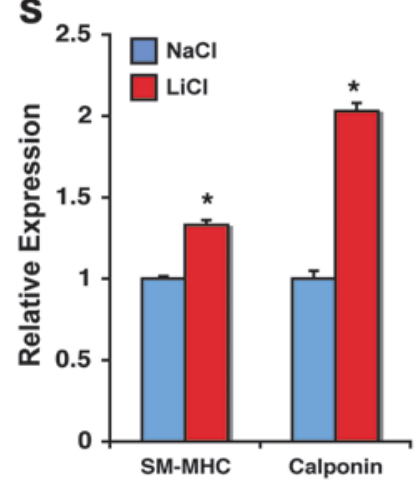

Figure 2

Wnt7b and $\beta$-catenin signaling regulates Pdgfr $\alpha$ and Pdgfr $\beta$ expression in the lung. (A-D) Immunostaining for Pdgfr $\beta$ expression in the developing lungs of wild-type ( $\mathbf{A}$ and $\mathbf{C}$ ) and Wnt7 b/acZ-null mutants (B and $\mathbf{D})$ at E10.5 (A and $\mathbf{B})$ and in developing blood vessels (arrows) at E12.5 (C and D). (E) Q-PCR for Pdgfr $\alpha$ and Pdgfr $\beta$ expression at E10.5. (F-I) Immunostaining for Pdgfr $\alpha$ expression in the developing lungs of wild-type $(\mathbf{F}$ and $\mathbf{H})$ and $W n t 7 b^{l a c z}$-null mutants $(\mathbf{G}$ and $\mathbf{I})$ at E10.5 (F and $\left.\mathbf{G}\right)$ and E12.5 (H and $\left.\mathbf{I}\right)$. (J) BAT-GAL Wnt reporter activity at E10.5 in the mesenchyme of the lung in an overlapping region where Pdgfr $\beta$ expression was observed. (K and $\mathbf{L})$ Immunostaining for Pdgfr $\beta$ expression in wild-type (K) and SM22 $\alpha$-cre:Ctnnb1floxfllox mutants (L) at E12.5. (M) Q-PCR for Pdgfr $\alpha$ and Pdgfr $\beta$ expression in wild-type and SM22 $\alpha$-cre:Ctnnb1 $1^{\text {floxflfox }}$ mutants at E10.5. (N) Q-PCR for axin2, Pdgfra, and Pdgfr $\beta$ expression from the lung buds of NaCl- and LiCl-treated embryos at E10.5. (O-R) SM22 $\alpha$ immunostaining in lungs of $\mathrm{NaCl}$ - and LiCl-treated E15.5 embryos. (S) Q-PCR for SM-MHC and calponin expression from the lung buds of $\mathrm{NaCl}$ - and $\mathrm{LiCl}$-treated embryos at E14.5. ai, airway. ${ }^{*} P<0.01$. Scale bars: $250 \mu \mathrm{m}(\mathbf{A}-\mathbf{D}, \mathbf{F}, \mathbf{G}$, and $\mathbf{J}), 150 \mu \mathrm{m}$ $(\mathbf{H}, \mathbf{I}, \mathbf{K}$, and L), $100 \mu \mathrm{m}(\mathbf{O}-\mathbf{R})$. 

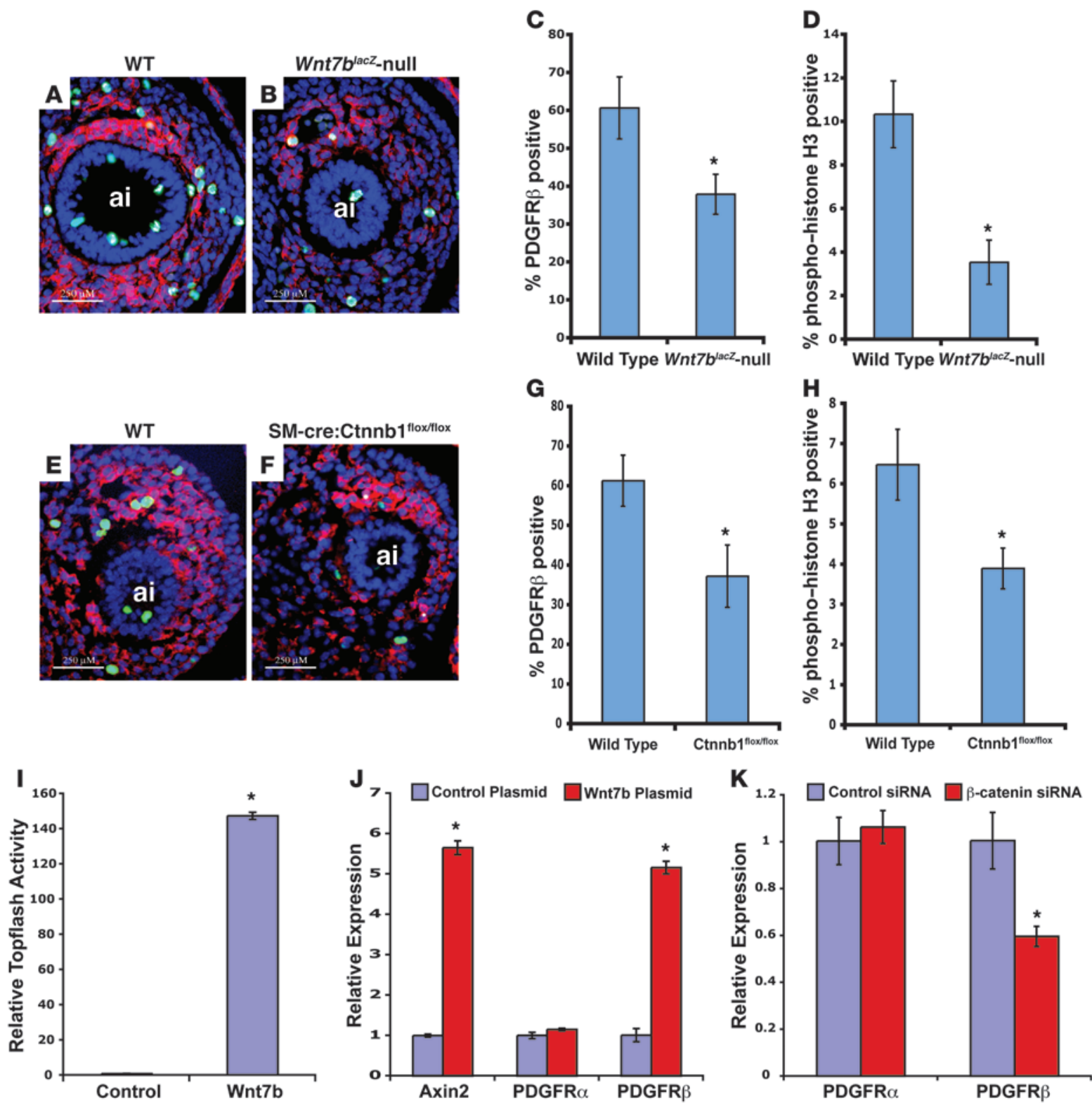

Figure 3

Wnt7b regulates proliferation of Pdgfr $\beta$-expressing SMC precursors, and $\beta$-catenin specifically regulates Pdgfr $\beta$ versus Pdgfr $\alpha$ expression in VSMCs. (A and B) Immunostaining for Pdgfr $\beta$ (red) and $\mathrm{PO}_{4}-\mathrm{H} 3$ (green) expression in wild-type (A) and Wnt7 $\mathrm{b}^{\prime a c Z}$-null mutants (B) at E10.5. (C and D) The percentage of Pdgfr $\beta$-expressing cells $(\mathbf{C})$ as well as proliferating lung mesenchyme was significantly reduced in $W n t 7 b^{\text {lacZ }}$-null mutants

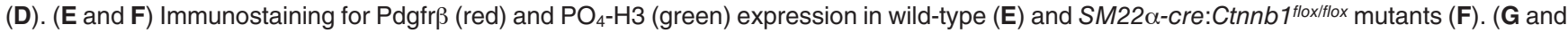
H) The percentage of Pdgfr $\beta$-expressing cells $(\mathbf{G})$ as well as proliferating lung mesenchyme was significantly reduced in SM22 $\alpha$-cre:Ctnnb $1^{\text {floxtflox }}$ mutants $(\mathbf{H})$ at E10.5. DAPI was used a counterstain to detect cell nuclei. (I) Wnt7b activated the SuperTOPFLASH Wnt reporter in Pac1 cells. (J) Wnt7b activated expression of Pdgfr $\beta$ and axin2 but not Pdgfr $\alpha$ in Pac1 cells. (K) siRNA-mediated knockdown of $\beta$-catenin in Pac1 cells led to decreased Pdgfr $\beta$ but not Pdgfr $\alpha$ expression. ${ }^{\star} P<0.001$. Scale bars: $250 \mu \mathrm{m}$.

SM-MHC was downregulated relative to those of heterozygous controls (Figure 1I). These data demonstrate that smooth muscle development in the lung requires $W n t 7 b$ and $\beta$-catenin signaling and suggests that the affects of Wnt7b on smooth muscle development are mediated by canonical Wnt signaling.

Smooth muscle precursor development requires Wnt7b and $\beta$-catenin signaling in the lung. Given the significant affect that loss of Wnt7b has on smooth muscle development early in lung development, we sought to determine whether smooth muscle precursors are affected in Wnt7b mutant lungs. Control and Wnt $7 b^{\text {lacZ }}$ mutant lung buds isolated at E10.5 were immunostained to detect Pdgfr $\beta$, which is highly expressed in vascular smooth muscle precursors and was required for their proliferation and migration (13). These analyses show that the numbers of Pdgfr $\beta$ - and Pdgfr $\alpha$-positive cells surrounding the epithelium of the early lung bud were significantly reduced in Wnt7b mutant lung buds compared with con- 
Table 1

Genes regulated by Wnt7b expression in Pac1 cells

\begin{tabular}{|c|c|c|c|c|}
\hline Gene symbol & Gene title & Fold change ${ }^{A}$ & Public ID & Q-PCR verified \\
\hline \multicolumn{5}{|c|}{ Genes implicated in canonical Wnt signaling } \\
\hline Axin2 & Axin 2 & 12.56 & NM_024355 & Yes \\
\hline Nkd2 & Naked homolog 2 (Drosophila) & 3.01 & Al072336 & Yes \\
\hline Tcf7 & T cell-specific transcription factor 7 & 2.80 & AW251860 & No \\
\hline Sfrp4 & Secreted frizzled-related protein 4 & 2.78 & AF140346 & Yes \\
\hline \multicolumn{5}{|c|}{ Genes implicated in vascular remodeling and smooth muscle development } \\
\hline Irx3 & Iroquois related homeobox 3 (Drosophila) & 3.72 & Al030203 & No \\
\hline Tnc & Tenascin C & 3.02 & Al176034 & Yes \\
\hline Alcam & Activated leukocyte cell adhesion molecule & 2.58 & NM_031753 & Yes \\
\hline Prrx1 & Paired related homeobox 1 & 2.48 & AA957064 & Yes \\
\hline Timp3 & Tissue inhibitor of metalloproteinase 3 & 2.18 & AA893169 & Yes \\
\hline Vegfd & Vascular endothelial growth factor D & 2.14 & Al170324 & Yes \\
\hline Edn1 & Endothelin 1 & 2.04 & NM_012548 & Yes \\
\hline Twist1 & Twist homolog 1 (Drosophila) & 2.04 & BF420349 & No \\
\hline Tgfb2 & Transforming growth factor_2 & 2.04 & NM_031131 & No \\
\hline
\end{tabular}

ARelative to mock-transfected Pac1 cells.

trols (Figure 2, A-D and F-I). There was no significant difference in apoptosis in wild-type or $W n t 7 b^{l a c Z}$ mutant lung buds, indicating that the decrease in Pdgfr $\beta$-positive cells was not due to cell death (Supplemental Figure 2). Moreover, Q-PCR analysis showed that expression of both Pdgfr $\alpha$ and Pdgfr $\beta$ was reduced in Wnt $7 b^{\text {lac } Z}$ mutant lung buds, indicating that expression of both receptors was reduced by loss of Wnt7b expression (Figure 2E).

BAT-GAL Wnt reporter mice revealed canonical Wnt activity in mesenchyme adjacent to airway epithelium at E10.5 in a pattern overlapping with Pdgfr $\alpha$ and Pdgfr $\beta$ expression (Figure 2J). To determine whether $\beta$-catenin was required for the expression of Pdgfr $\alpha$ and Pdgfr $\beta$ in the early lung, E12.5 SM22 $\alpha$-cre:Ctnnb1 $1^{\text {flox/flox }}$ mutant and control lungs were immunostained for Pdgfr $\beta$ expression. In control lungs, Pdgfr $\beta$ protein was broadly expressed throughout the mesenchyme, with the highest levels of staining found close to the airway epithelium, coalescing around developing blood vessels (Figure 2K). In contrast, both the intensity and breadth of Pdgfr $\beta$ staining was drastically reduced in SM22 $\alpha$-cre:Ctnnb $1^{\text {flox } f l o x}$ mutant lungs (Figure 2L). Q-PCR analysis showed that expression of both Pdgfr $\alpha$ and Pdgfr $\beta$ was significantly downregulated in $S M 22 \alpha-c r e: C t n n b 1^{\text {flox flox }}$ mutants (Figure $2 \mathrm{M}$ ).

To determine whether $\mathrm{Wnt} / \beta$-catenin signaling was sufficient to promote smooth muscle precursor development in the lung, mouse embryos were treated with either $\mathrm{NaCl}$ or $\mathrm{LiCl}$ during embryogenesis. $\mathrm{LiCl}$ inhibits the kinase activity of Gsk3 $\beta$, and previous studies have shown that systemic treatment of developing embryos in utero with $\mathrm{LiCl}$ activates $\mathrm{Wnt} / \beta$-catenin signaling in vivo (14-16). A 2.5-fold increase in axin 2 expression was observed in the lung of LiCl-treated embryos relative to $\mathrm{NaCl}$-treated embryos, indicating activation of canonical Wnt signaling (Figure $2 \mathrm{~N}$ ). Remarkably, Pdgfr $\beta$ and Pdgfro expression in the lung was dramatically increased in LiCl-treated embryos relative to $\mathrm{NaCl}$ treated embryos, indicating that Wnt $/ \beta$-catenin signaling activates expression of these receptors (Figure $2 \mathrm{~N}$ ). In addition, airway and vascular smooth muscle layers were thicker and expression levels of SM-MHC and calponin-h1 were increased in LiCl-treated embryos,
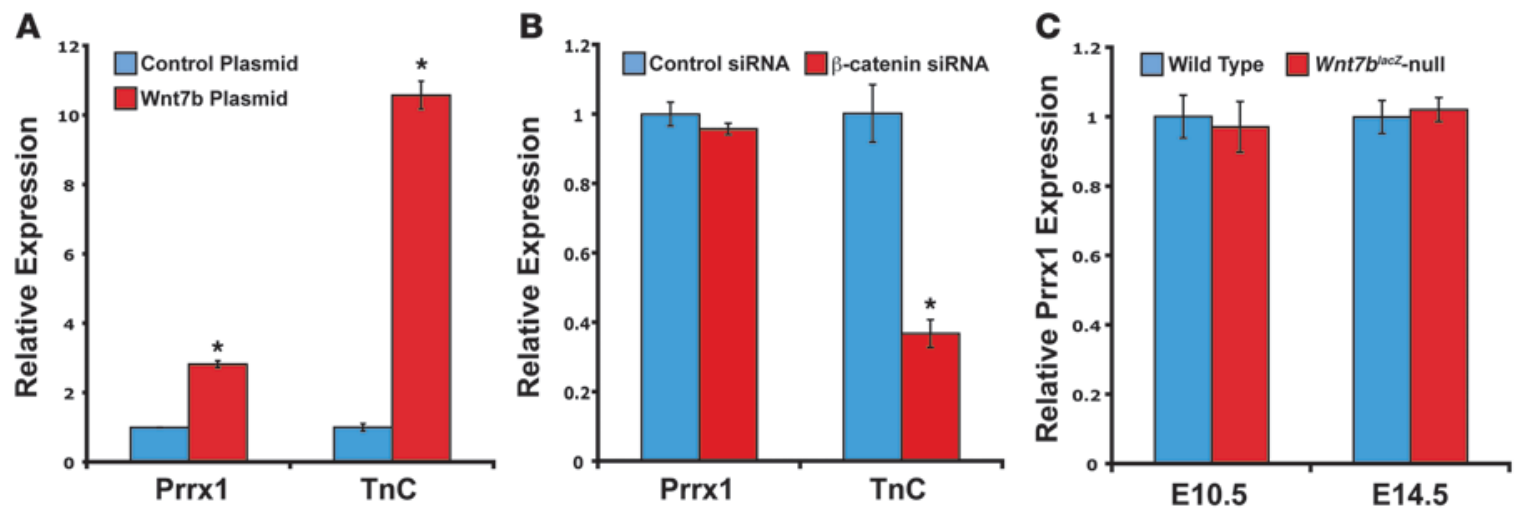

Figure 4

Wnt/ $\beta$-catenin is necessary and sufficient for Tnc expression. (A) Wnt7b expression activated Prrx1 and Tnc expression in Pac1 cells. (B) siRNA-mediated knockdown of $\beta$-catenin expression led to decreased Tnc but not Prrx1 expression. (C) Prrx1 expression was unchanged in Wnt7 b bacZ-null mutants at both E10.5 and E14.5. ${ }^{*} P<0.01$. 

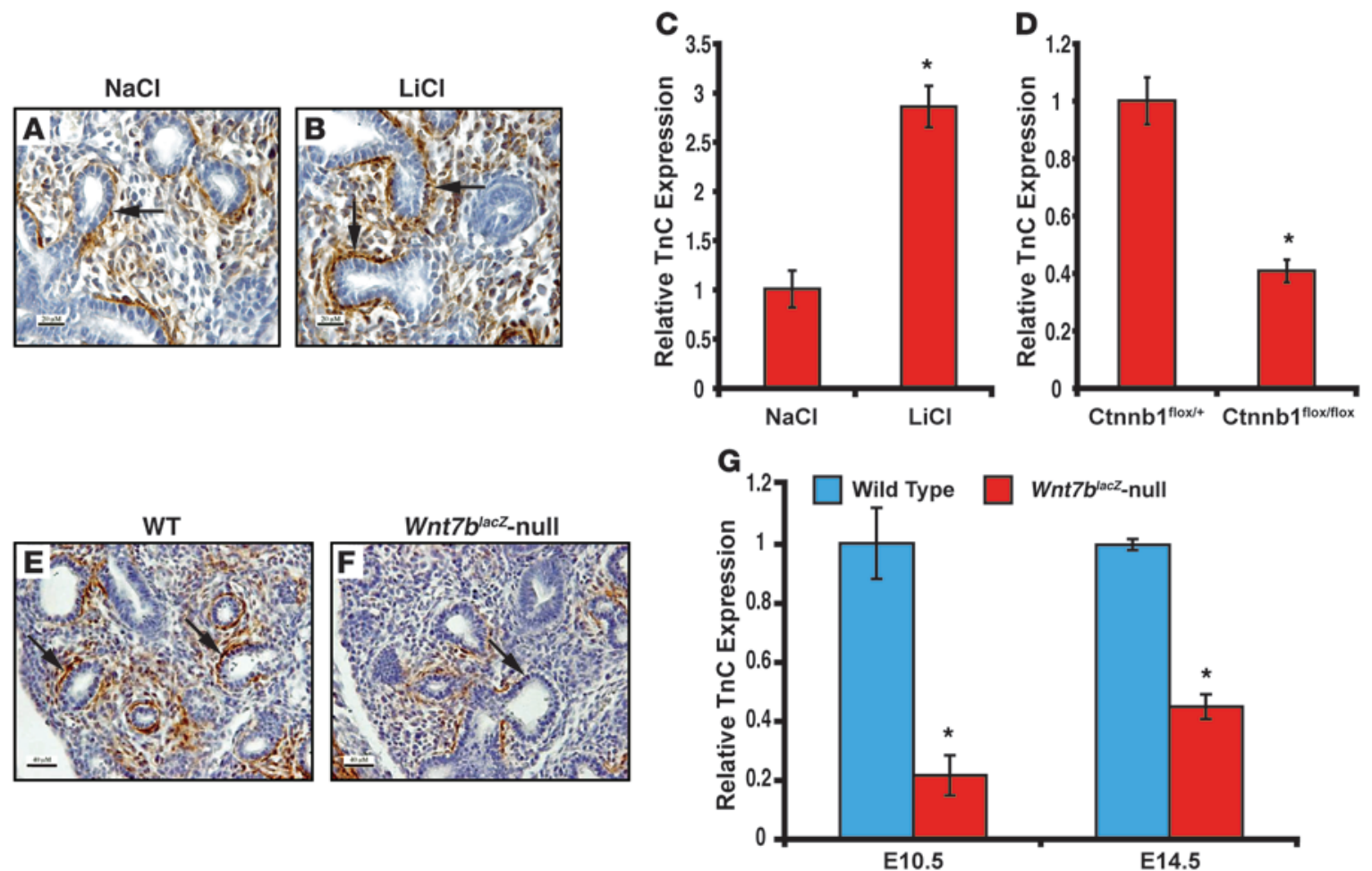

\section{Figure 5}

$\beta$-Catenin and Wnt7b signaling regulate Tnc expression in vivo. (A and B) Immunostaining for Tnc expression in NaCl- and LiCl-treated wildtype embryos at E15.5 showed increased Tnc expression (arrows) surrounding the airways of LiCl-treated embryos. (C) Expression of Tnc was induced approximately 3-fold by LiCl treatment. (D) Loss of $\beta$-catenin expression in SMC precursors in SM22 $\alpha$-cre:Ctnnb $1^{\text {floxfflox }}$ mutants resulted in a quantitative loss of Tnc expression in vivo at E10.5. (E-G) Tnc expression was significantly reduced in Wnt7 $b^{\text {lacZ }}$-null mutants as assessed by immunohistochemistry at E14.5 (E and F) and quantitatively by Q-PCR (G). ${ }^{*} P<0.001$. Scale bars: $20 \mu \mathrm{m}(\mathbf{A}$ and $\mathbf{B}), 40 \mu \mathrm{m}(\mathbf{E}$ and $\mathbf{F})$.

indicating that Wnt $/ \beta$-catenin signaling is sufficient to promote smooth muscle development in the lung (Figure 2, O-S).

$W n t 7 b$ and $\beta$-catenin signaling regulate Pdgfr $\beta$ expression in VSMC. We and others have previously shown that Wnt7b is required for mesenchymal proliferation during lung development $(8,17)$. The loss of Pdgfr $\beta$ in Wnt7b mutants may therefore reflect either a requirement for $W n t 7 b$ signaling in the expansion of Pdgfr $\beta$-expressing smooth muscle precursors in the surrounding mesenchyme or a role in their specification and differentiation. Costaining for phospho-histone $\mathrm{H} 3\left(\mathrm{PO}_{4}-\mathrm{H} 3\right)$ and $\mathrm{Pdgfr} \beta$ protein expression revealed that loss of either Wnt7b or $\beta$-catenin leads to dramatic reductions in the proliferation of Pdgfr $\beta$-expressing cells (Figure 3, A-H), suggesting that reduced proliferation is at least partially responsible for the loss of SMC precursors in Wnt7b mutant lungs.

In addition to the reduced proliferation of Pdgfr $\beta$-expressing cells, the overall level of Pdgfr $\beta$ expression in each cell also appeared to be lower in Wnt $7 b^{\text {lac Z }}$ and $S M 22 \alpha$-cre:Ctnnb1 flox/flox mutants, suggesting that Wnt signaling may control Pdgfr $\beta$ expression in SMC precursors. To assess this possibility, Wnt7b was overexpressed in the pulmonary VSMC line Pac1 and levels of Pdgfr $\beta$ expression were determined by Q-PCR. Of note, expression of Wnt7b was sufficient to activate canonical Wnt signaling in Pac1 cells, as demonstrated by increased activation of a Wnt/ $\beta$-catenin reporter and increased axin 2 expression relative to control transfected cells (Figure 3, I and J). Expression of Wnt7b was sufficient to induce Pdgfr $\beta$ expression in Pac1 cells (Figure 3J).
Furthermore, siRNA-mediated inhibition of $\beta$-catenin expression led to a significant reduction in $\operatorname{Pdgfr} \beta$ expression (Figure $3 \mathrm{~K})$. Although Pdgfr $\beta$ expression responded specifically to Wnt7b and $\beta$-catenin signaling, ChIP did not reveal a direct association of $\beta$-catenin with conserved TCF binding elements (TBEs) in the mouse Pdgfr $\beta$ promoter (data not shown) (18). Moreover, expression of neither Wnt7b nor $\beta$-catenin siRNA affected Pdgfr $\alpha$ expression in Pac1 cells, which may correlate with the vascular lineage of this cell line (Figure 3, J and K). These data suggest that Wnt7b regulates overall lung mesenchymal proliferation, including Pdgfr $\beta$ positive smooth muscle precursors, but this effect may be indirect and transmitted through additional pathways.

Wnt7b is necessary and sufficient for Tnc expression in VSMCs and in lung development. The regulation of Pdgfr $\beta$ expression by Wnt7b signaling raised the possibility that $\mathrm{Wnt} 7 \mathrm{~b}$ might regulate a broad transcriptional program critical for smooth muscle development. We therefore compared the gene expression profiles of Pac1 cells expressing Wnt7b and non-expressing cells using microarray differential gene expression analysis. Several known targets of Wnt signaling including axin 2 and naked 2 were upregulated in Pac1 cells expressing Wnt7b (Table 1). Interestingly, multiple genes implicated in smooth muscle development were also upregulated in Wnt7bexpressing Pac1 cells, including the paired-homeobox transcription factor Prrx1 and the ECM protein Tnc, both of which have been shown to promote smooth muscle growth and differentiation and are required for proper lung development (Table 1) (19-22). To con- 
A

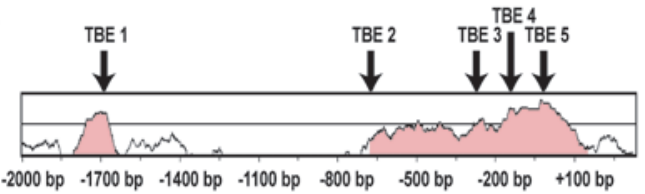

B

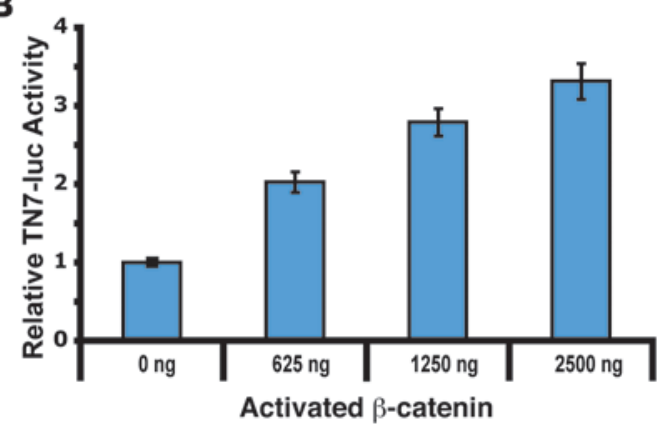

C

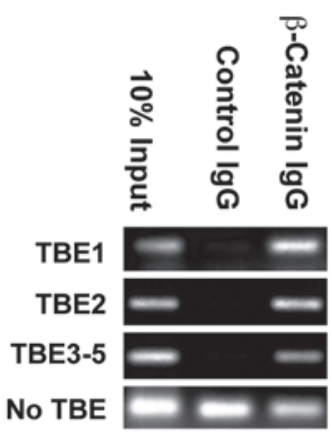

D

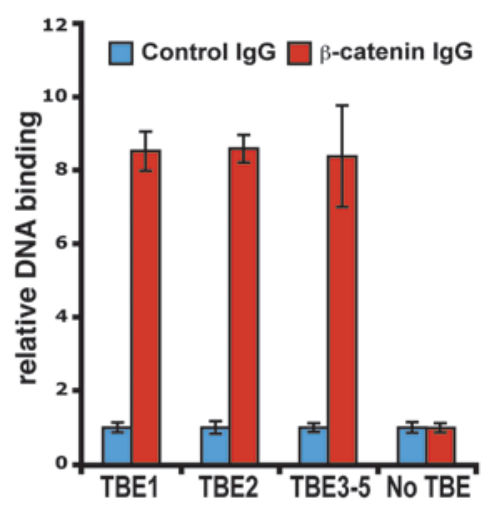

Figure 6

Tnc is a direct target of Wnt signaling in the lung. (A) The proximal Tnc mouse promoter region contains 5 TBEs. (B) The pTnc.luc reporter was activated in a dose-dependent manner by expression of an activated form of $\beta$-catenin in Pac1 cells. (C and D) ChIP assays using agarose gel electrophoresis (C) and Q-PCR (D) showed that $\beta$-catenin was associated with the TBE1, TBE2, and TBE3-5 regions in the mouse Tnc promoter in the lung.

firm that Wnt7b signaling was sufficient to activate these genes in Pac1 cells, we examined the mRNA levels for Prrx1 and Tnc in control and Wnt7b-transfected cells by Q-PCR. Prrx1 and Tnc were both significantly upregulated in Wnt7b-transfected cells relative to control (Figure 4A). Previous studies have shown that Prrx1 can directly activate Tnc (19). Thus, Wnt signaling could directly activate Tnc or activate Tnc through direct activation of Prrx1. To discern between these two possibilities, we examined mRNA levels for Prrx1 and Tnc in Pac1 cells transfected with either control or $\beta$-catenin siRNA. While Tnc mRNA levels were significantly reduced in $\beta$-catenin siRNA-treated Pac1 cells, Prrx1 expression was unaffected (Figure 4B). In agreement with these data, Prrx1 levels were unaffected by the loss of Wnt7b in vivo (Figure 4C).

In order to determine whether activating canonical Wnt signaling induces Tnc expression in vivo, immunostaining for Tnc expression was performed on the lungs of $\mathrm{NaCl}$ - and LiCl-treated embryos. Tnc staining was significantly increased in the lungs of LiCl-treated embryos using both immunostaining and Q-PCR analysis (Figure 5, A-C). To determine whether $\beta$-catenin signaling was required for Tnc expression during lung development, Q-PCR was used to compare Tnc expression in SM22 $\alpha$ :Ctnnb $1^{\text {flox/flox }}$ and control embryos. Again, consistent with the results obtained above, the levels of Tnc mRNA were significantly reduced in the

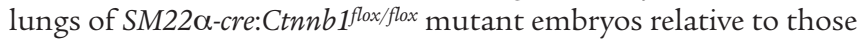
of control embryos (Figure 5D). Finally, to determine whether Wnt7b was required for Tnc expression during lung development, immunostaining and Q-PCR analysis for Tnc expression was performed on control and $W n t 7 b^{\text {lac }}$ mutants. Tnc expression was significantly reduced in $W n t 7 b^{l a c Z}$ mutants lungs relative control (Figure 5, E-G). Together, these data indicate that Wnt7b signaling via the canonical Wnt/ $\beta$-catenin pathway is necessary and sufficient for Tnc expression during lung development.

Tnc is a direct target of Wnt/ $\beta$-catenin signaling and regulates $P d g f r \alpha$ and Pdgfr $\beta$ expression in the lung. Comparative genomic analysis between the mouse and human TNC genes identified 5 conserved
TBEs located within a $2-\mathrm{kb}$ region upstream of the transcriptional start site (Figure 6A). One of these sites, TBE5, is located within a previously described Tnc promoter construct, pTnc.luc (23). To determine whether this site was responsive to $\beta$-catenin transactivation, Pac1 cells were transfected with the pTnc.luc reporter along with an expression plasmid encoding an activated form of $\beta$-catenin. These studies showed that expression of activated $\beta$-catenin trans-activates the pTnc.luc reporter in a dose-dependent manner (Figure 6B). ChIP assays were performed on chromatin obtained from E12.5 lung tissue using an anti- $\beta$-catenin antibody to determine whether $\beta$-catenin associated with any of the $5 \mathrm{TBE}$ sites in the $2-\mathrm{kb}$ mouse Tnc promoter. ChIP analysis showed that $\beta$-catenin associates with all 5 TBEs in the proximal $2-\mathrm{kb}$ Tnc mouse promoter (Figure $6, \mathrm{C}$ and D). In contrast, $\beta$-catenin binding was not enriched in an unrelated region of the genome upstream of the VegfD locus (Figure 6, C and D).

Tnc has previously been shown to be required for Pdgfro expression in glomerular mesangial cells (13), raising the possibility that the regulation of Pdgfr $\alpha$ and Pdgfr $\beta$ expression by Wnt7b and $\beta$-catenin signaling during lung development shown in Figure 2 may be mediated by its ability to regulate Tnc. This hypothesis is concordant with the lack of evidence supporting a direct regulation of the Pdgfr $\beta$ promoter by Wnt $/ \beta$-catenin signaling (data not shown). To test this hypothesis, E11.5 lung buds were cultured for 48 hours in the presence of either an antibody that blocks Tnc function (Tnc IgG) or a nonspecific control antibody (control IgG). In control IgG-treated explanted lung buds, high levels of Pdgfr $\beta$ expression were found in mesenchymal cells adjacent to airway epithelium at the periphery of the explant, which corresponds to the distal region of the developing lung in vivo (Figure 7A). In contrast, both the number of cells expressing Pdgfr $\beta$ and the intensity of their expression were reduced in Tnc IgG-treated explants (Figure 7B). Q-PCR analysis revealed that the levels of both Pdgfra and Pdgfrb mRNA were significantly reduced in Tnc IgG-treated explants relative to controls (Figure 7C). Moreover, 
control IgG

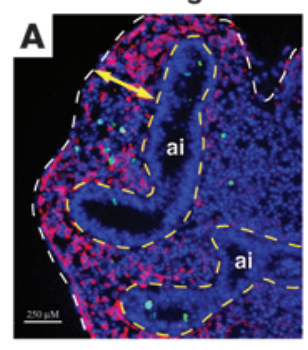

E

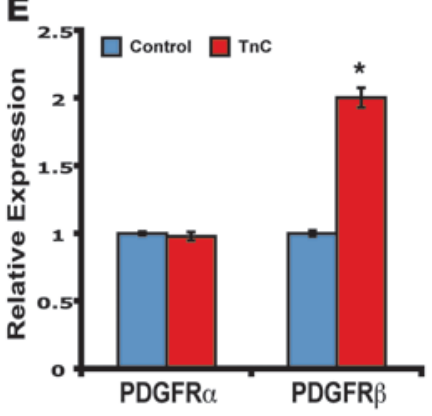

TnC IgG

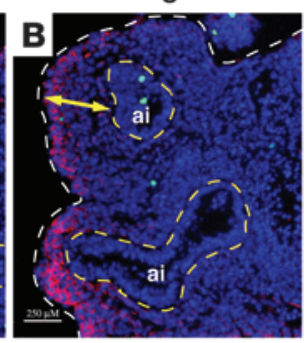

$\mathbf{F}$

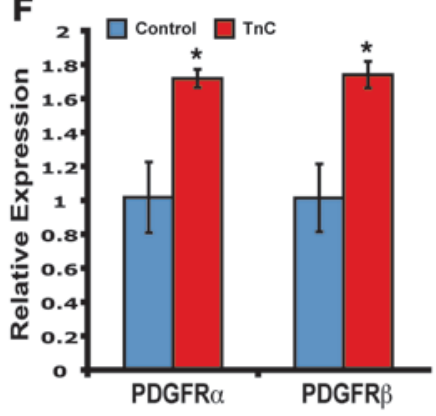

C

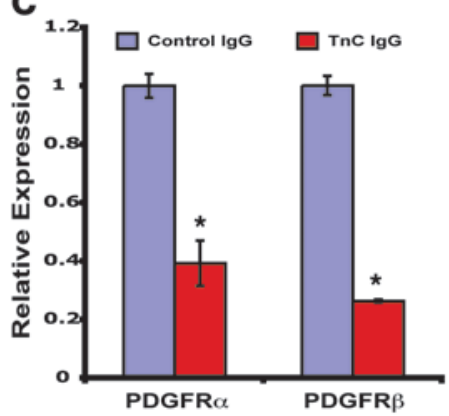

G
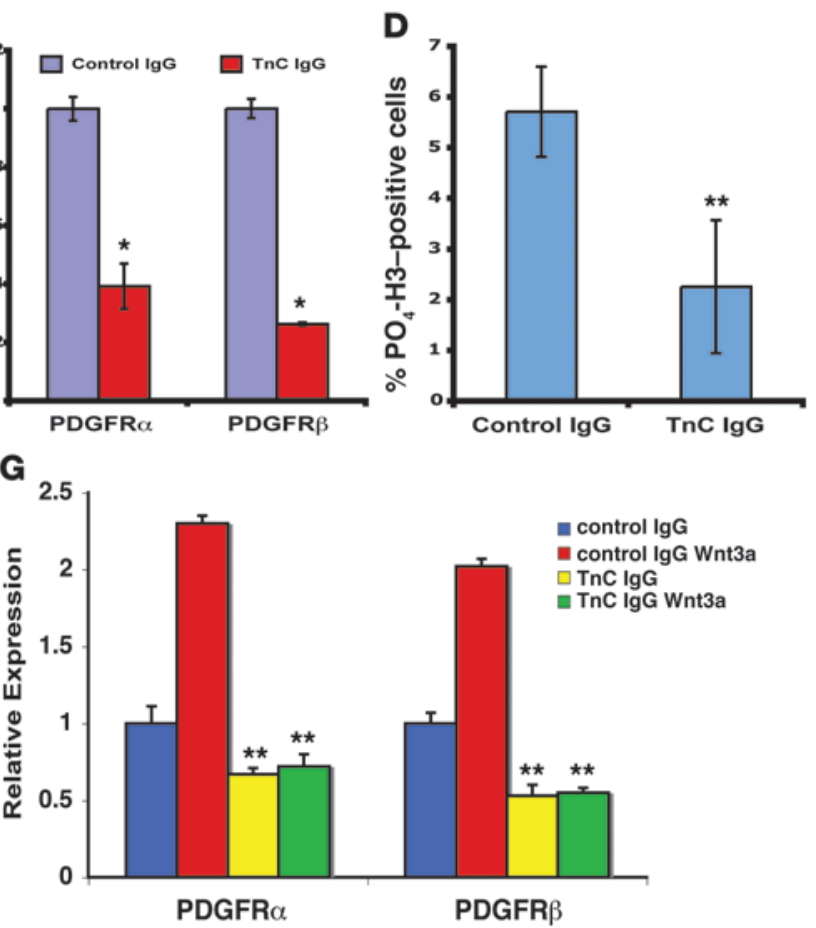
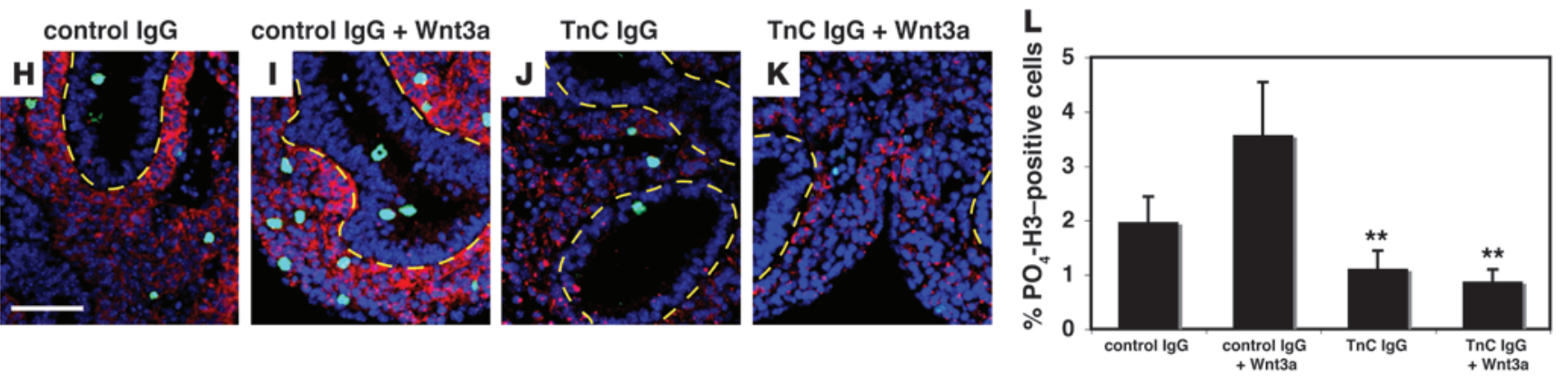

Figure 7

Tnc expression directly modulates Pdgfr $\beta$ expression. (A-C) Treatment of lung explants from E11.5 embryos with a Tnc-blocking antibody reduced Pdgfr $\beta$ expression (arrows) by immunohistochemistry (A and $\mathbf{B}$ ) and reduced both Pdgfr $\alpha$ and Pdgfr $\beta$ expression by Q-PCR (C). The white dotted lines in A and $\mathbf{B}$ denote the external outline of lung explant; yellow dotted lines denote developing airways. (D) Antibody-mediated blocking of Tnc function reduced lung mesenchymal proliferation. (E) Tnc protein treatment of Pac-1 cells led to increased Pdgfr $\beta$ but not Pdgfr $\alpha$ expression by Q-PCR. (F) Tnc protein treatment of E11.5 mouse lung explants resulted in increased expression of both Pdgfr $\alpha$ and Pdgfr $\beta$ as detected by Q-PCR. (G-K) Treatment of lung explants with Wnt3a and either non-immune IgG (control lgG) or Tnc-blocking antibody (Tnc lgG) showed that blocking Tnc decreased Wnt3a-induced Pdgfr $\alpha$ and Pdgfr $\beta$ expression, as assessed by Q-PCR (G) and immunohistochemistry $(\mathbf{H}-\mathbf{K})$. (H-L) Blocking Tnc also inhibited the Wnt3a-induced increase in proliferation observed in lung explants as noticed by $\mathrm{PO}_{4}-\mathrm{H} 3$ immunostaining (green nuclei). ${ }^{\star} P<0.001 ;{ }^{* *} P<0.03$. Scale bars: $250 \mu \mathrm{m}$.

proliferation of Pdgfr $\beta$-expressing cells was significantly reduced in Tnc IgG-treated explants relative to those treated with control antibody (Figure 7D).

To determine whether Tnc was sufficient to induce Pdgfr expression, Pac1 cells were treated with purified Tnc protein and expression of Pdgfr $\alpha$ and Pdgfr $\beta$ was assessed. Expression of Pdgfr $\beta$ was significantly higher in Tnc-treated Pac1 cells relative to control cells (Figure 7E). In contrast, Tnc did not increase Pdgfr $\alpha$ expression in Pac1 cells, which is consistent with their vascular origin (Figure 7E). To determine whether Tnc was sufficient to increase Pdgfr $\alpha$ and Pdgfr $\beta$ signaling in developing lung tissue, E11.5 lung buds were cultured for 3 days in either control media or media containing purified Tnc protein. Expression of both Pdgfr $\alpha$ and Pdgfr $\beta$ was significantly higher in Tnc-treated explants than in control explants (Figure 7F).

Finally, to determine whether the regulation of Pdgfr $\alpha$ and Pdgfr $\beta$ by Wnt signaling was dependent upon Tnc activity, we treated lung explants with recombinant Wnt3a and either a control antibody or the Tnc-blocking antibody and assessed Pdgfr $\alpha$ and Pdgfr $\beta$ expression by immunostaining and Q-PCR and proliferation by $\mathrm{PO}_{4}-\mathrm{H} 3$ immunostaining. As expected, treatment of lung explants with Wnt3a increased Pdgfr $\alpha$ and Pdgfr $\beta$ expression in control IgG-treated explants (Figure 7, G-I). In contrast, Pdgfr $\alpha$ and Pdgfr $\beta$ expression was significantly reduced in Wnt3a/Tnc blocking antibody-treated explants (Figure 7, G-K). Moreover, proliferation in Tnc-treated explants was reduced compared with 

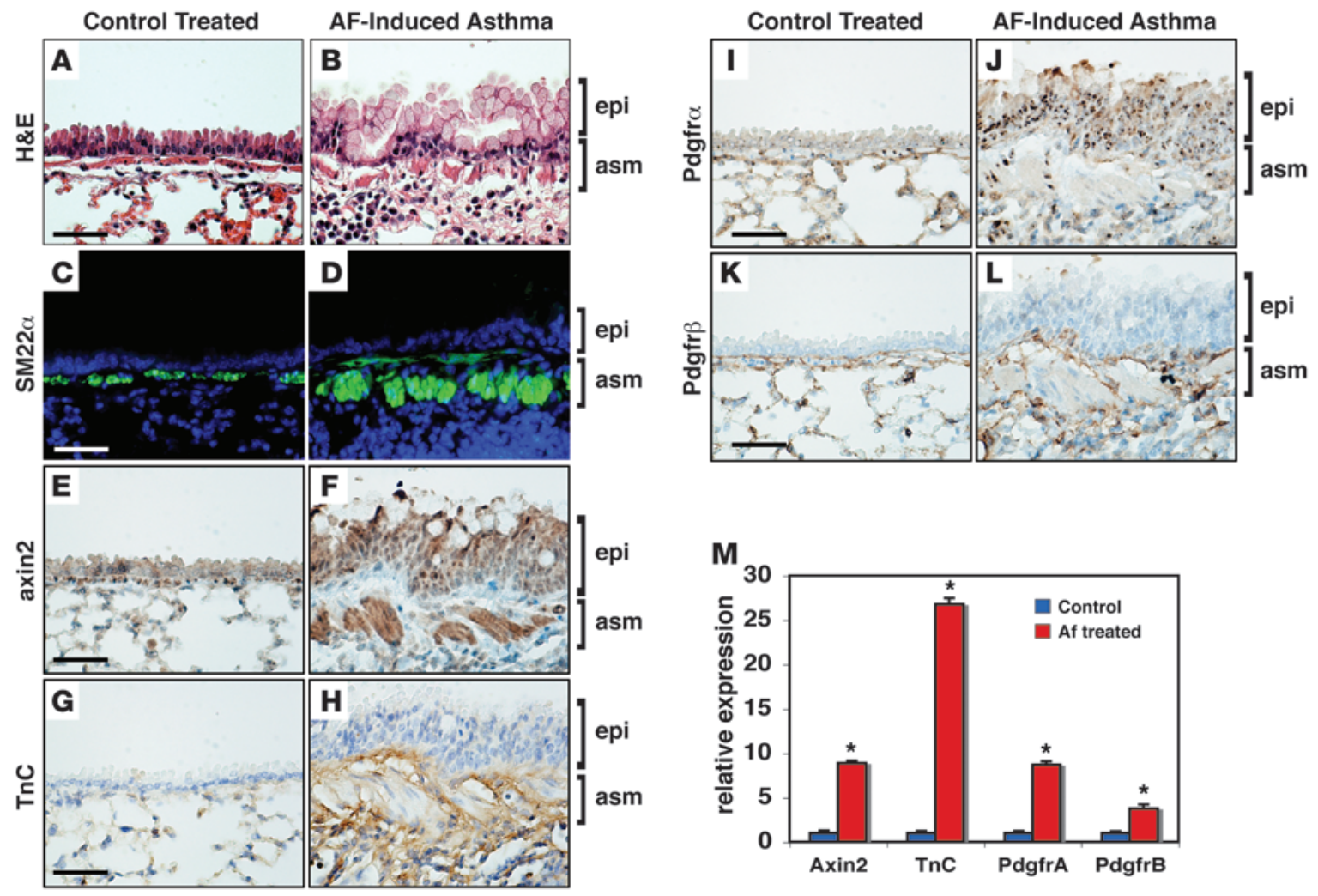

Figure 8

Increased expression of the $\mathrm{Wnt} / \mathrm{Tnc} / \mathrm{Pdgfr}$ pathway in $A$. fumigatus-induced asthma in mice. (A-L) Mice treated with $A$. fumigatus to induce airway hyperresponsiveness and inflammation showed increased smooth muscle mass as assessed by H\&E staining (A and B) and SM22 $\alpha$ immunohistochemistry (C and $\mathbf{D})$. Coincident with this reaction, increased expression of axin2 (E and $\mathbf{F})$, Tnc $(\mathbf{G}$ and $\mathbf{H})$, Pdgfra (I and $\mathbf{J})$, and Pdgfr $\beta$ (K and L) were observed. (M) Q-PCR was used to quantitate the increase in expression of axin2, Tnc, Pdgfr $\alpha$, and Pdgfr $\beta .{ }^{*} P<0.001$. Epi, epithelium; asm, airway smooth muscle. Scale bars: $100 \mu \mathrm{m}$.

controls (Figure 7, H-L). These data show that the increase in Pdgfr $\alpha$ and Pdgfr $\beta$ as well as the increase in proliferation by Wnt in the lung is dependent on Tnc activity.

Increased Wnt/Tnc/Pdgfr expression in a mouse model of asthma and in buman pulmonary bypertension patients. To determine whether expression of this pathway was altered in adult smooth muscle-associated lung diseases, we examined expression and activity of the Wnt, Tnc, and Pdgfr components in a mouse model of allergen-induced asthma and samples from human patients with pulmonary arterial hypertension (PAH). The Aspergillus fumigatus-induced airway asthma model mimics human asthma by inducing airway smooth muscle proliferation, leading to increased airway smooth muscle (ASM) mass (24). As shown in Figure 8, increased ASM mass was observed in A. fumigatustreated animals (Figure 8, A-D). Moreover, increased expression of axin2, Tnc, Pdgfr $\alpha$, and Pdgfr $\beta$ was observed in the airway smooth muscle of A. fumigatus-treated animals (Figure 8, E-L). Expression of axin2 and Pdgfro was also upregulated in airway epithelium of A. fumigatus-treated animals (Figure 8, E, F, I, and J). Q-PCR showed that these increases were highly significant (Figure 8M).

Human PAH is characterized by a dramatic increase in pulmonary vascular smooth muscle proliferation and eventual narrowing of these vessels, leading to right ventricular overload in the heart and eventual heart failure (25). Histological analysis of human PAH samples showed increased levels of smooth muscle $\alpha$-actin and Tnc staining (Figure 9, A-H, K, and L), as has been previously described (23).
Remarkably, increased expression of axin2, Pdgfr $\alpha$, and Pdgfr $\beta$ was also observed in PAH samples (Figure 9, I, J, and M-P). Taken together, these data support a model in which Wnt signaling regulates both the expression and the number of Pdgfr $\alpha$ - and Pdgfr $\beta$-positive SMC precursors, in part through direct regulation of Tnc transcription, to promote SMC precursor proliferation and development in embryonic lung development as well as in adult lung disease (Figure 10).

\section{Discussion}

Defects in pulmonary smooth muscle development and function are leading contributors to numerous lung diseases, including pulmonary hypertension and asthma. Although signaling between airway epithelium and airway smooth muscle is critical for development of each of these cell lineages, little is understood about the relevance of these interactions in development and human disease. Expression of Tnc and Pdgfr $\beta$ is linked with several adult lung diseases, including asthma, pulmonary fibrosis, and pulmonary hypertension $(23,26-28)$. We show that paracrine Wnt signaling from the developing airway epithelium is required for expansion and development of smooth muscle precursors in the lung through a Wnt/Tnc/Pdgfr signaling axis. Moreover, we show that expression and activity of this pathway is increased in a mouse asthma model and in human PAH patients. Thus, our data establish the critical importance of a Wnt/Tnc/Pdgfr pathway in both lung development and adult lung disease. 

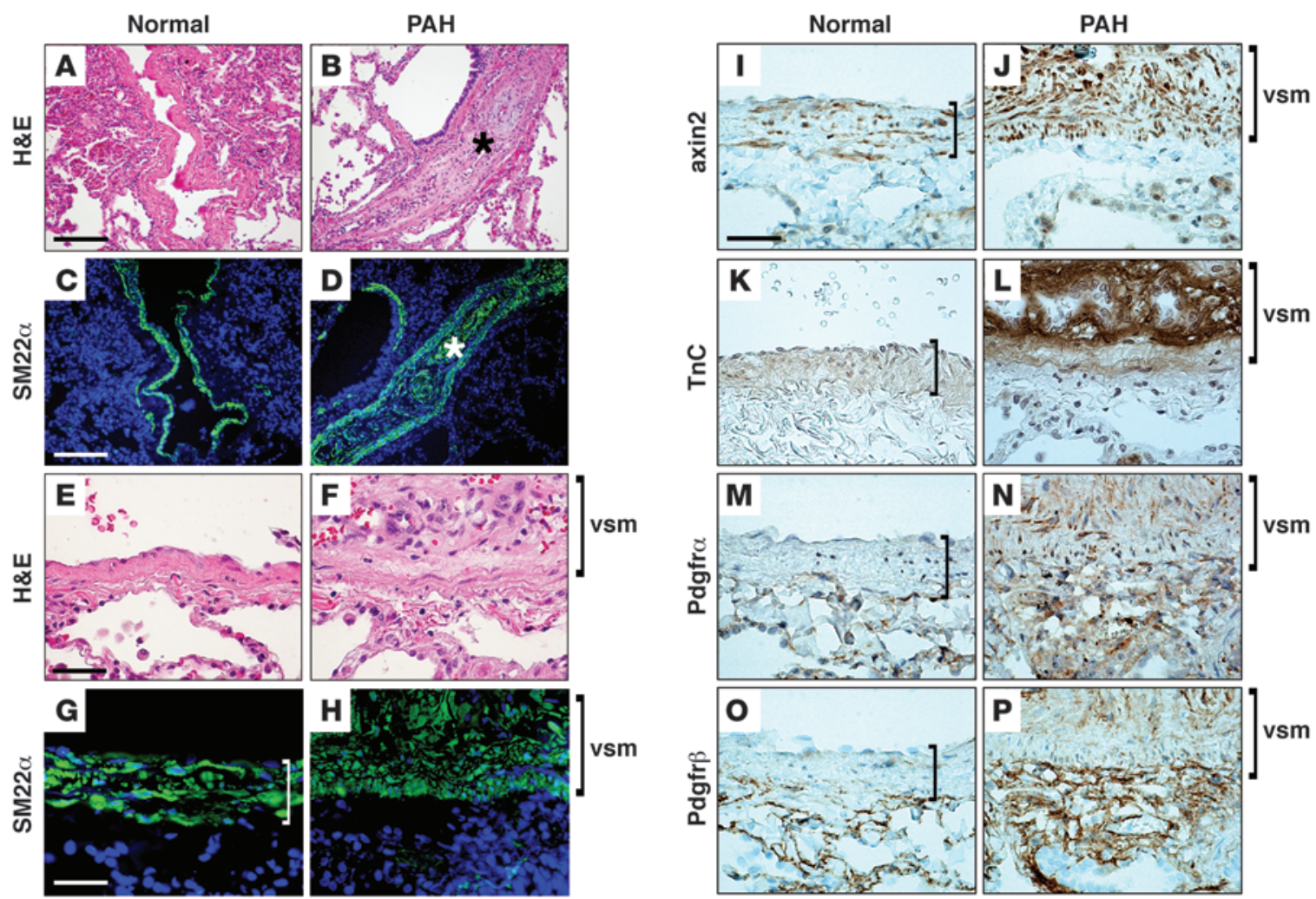

Figure 9

Increased expression of the Wnt/Tnc/Pdgfr pathway in pulmonary hypertension in humans. (A-H) Histological sections from human patients with $\mathrm{PAH}$ were stained with $\mathrm{H \& E}(\mathbf{A}, \mathbf{B}, \mathbf{E}$, and $\mathbf{F})$ or $\mathrm{SM} 22 \alpha(\mathbf{C}, \mathbf{D}, \mathbf{G}$, and $\mathbf{H})$ to detect vascular smooth muscle overgrowth in pulmonary arteries. (I-P) Expression of axin2 ( $\mathbf{I}$ and $\mathbf{J})$, Tnc ( $\mathbf{K}$ and $\mathbf{L})$, Pdgfr $\alpha(\mathbf{M}$ and $\mathbf{N})$, and $\mathbf{P d g f r} \beta$ ( $\mathbf{O}$ and $\mathbf{P})$ was increased in patients with PAH compared with normal controls. Vsm, vascular smooth muscle. Scale bars: $400 \mathrm{~mm}(\mathbf{A}-\mathbf{D}), 100 \mu \mathrm{m}(\mathrm{E}-\mathbf{P})$.

Many airway diseases in the lung are the result of environmental insults and the subsequent inflammatory response from these chronic injuries. Diseases such as pulmonary fibrosis, pulmonary hypertension, and asthma involve damage by prolonged exposure to environmental agents followed by successive rounds of inflammation and wound healing. During these processes, SMCs and myofibroblasts rapidly proliferate and expand to replace damaged tissue. However, expansion of these mesenchymal cell types and subsequent deposition of abnormal amounts of ECM proteins can become disruptive to airway and vascular function. Interestingly, Wnt7b and Tnc expression are both upregulated in mouse models of pulmonary fibrosis, and Wnt7b and Pdgfr $\beta$ are upregulated in some forms of human idiopathic pulmonary fibrosis and hypertension, respectively $(23,26-30)$. Moreover, polymorphisms in Tnc and Pdgfr $\beta$ have been linked to asthma, while Tnc mutations attenuate ovalbumin-induced asthma in mice including the associated increase in the numbers of bronchiolar goblet cells, a phenotype that can be mimicked by activating canonical Wnt signaling in the airway epithelium (31-34). Taken together, our results suggest that the Wnt/Tnc/Pdgfr signaling axis described in this paper may be a common link in human airway disease.

Our findings demonstrate that Tnc is a direct target of Wnt signaling in the lung and that loss of Tnc, and in turn Pdgfr $\beta$ expression, is in part responsible for the SMC defects observed in $W n t 7 b^{\text {lac }} Z_{-}$ null mutants. A previous report showed that decreased Tnc expression leads to SMC apoptosis (22). Thus, loss of Tnc might be a primary cause of the increased apoptosis observed in the VSMCs of Wnt7b-deficient animals (8). Other ECM proteins, including fibronectin $(\mathrm{Fn})$, have been shown to be targets of Wnt signaling (35). ECM proteins are critical regulators of stem/progenitor cell differentiation and act primarily through regulation of cell-ECM interactions, which helps create specific microenvironments or niches within tissues that are critical for stem/progenitor cell selfrenewal and differentiation. Given the role of Wnt signaling in the regulation of precursor and progenitor populations, governing the expression of ECM proteins is likely an important mechanism underlying the ability of Wnt signaling to regulate proliferation and differentiation of SMC precursors in the lung. Tnc likely acts cooperatively with other ECM proteins to promote proper SMC development in part through regulation of smooth muscle precursor expansion and differentiation. Interestingly, Tnc and Fn have been shown to cooperatively regulate SMC proliferation and migration, respectively, in models of pulmonary hypertension (20). Thus, the ability of Wnt signaling to regulate both Tnc and Fn reveals a critical role for this pathway not only in lung SMC development and diseases but also in SMC populations in other tissues.

Although our data demonstrate that Wnt7b plays an important role in the paracrine regulation of lung smooth muscle development, other Wnt ligands likely act redundantly with Wnt7b in this process. One such ligand, Wnt7a, is expressed in airway epithelium, but lung defects have not been reported (36). Importantly, we show that Wnt7a expression is significantly upregulated in the lungs of 


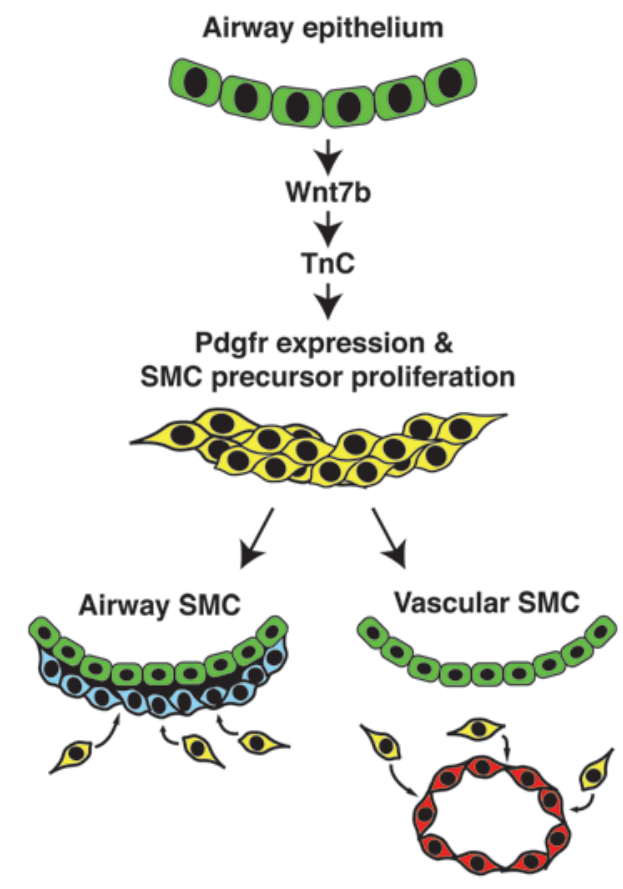

\section{Figure 10}

Model of a Wnt/Tnc/Pdgfr signaling axis regulating lung SMC development. Airway epithelium (green) expresses Wnt7b, which acts on mesenchymal SMC precursors (yellow). Wnt7b and $\beta$-catenin signaling directly activates Tnc expression and in turn promotes airway SMC (blue) and VSMC (red) precursor proliferation and development.

Wnt7 $b^{\text {lac }}$-null mutants. Mouse Wnt5a mutants exhibit defects in lung mesenchymal proliferation, although specific smooth muscle defects were not reported (37). Conversely, overexpression of Wnt5a leads to defects in lung vascular development through repression of Shh, which in turn leads to increased Fn expression (38). Since Wnt5a is a non-canonical Wnt and non-canonical signaling often antagonizes canonical signaling, these findings suggest that multiple Wnt ligands expressed in lung epithelium might act cooperatively to balance SMC differentiation in the lung. In addition to Wnt ligand redundancy, the source of SMC precursors has recently expanded to include mesothelial cell migration followed by epithelial to mesenchymal transformation to generate VSMCs in the lung (39). Thus, Wnt signaling may be important in orchestrating the complex series of developmental processes required for proper SMC development injury response in the lung.

Our findings showing that Wnt7b and canonical Wnt/ $\beta$-catenin signaling affects both smooth muscle development and survival provides a new and important pathway to target for therapeutic intervention in smooth muscle-dependent lung diseases including asthma and pulmonary hypertension. Wnt signaling is under intensive investigation as a target for anticancer drugs, and therapies derived from these studies may be useful in treating pulmonary diseases, especially ones characterized by excessive smooth muscle proliferation.

\section{Methods}

Animals. Generation and genotyping of the Wnt7 $7 b^{\text {lacZ }}$, Ctnnb $1^{\text {flox } / f l o x}, B A T-G A L$, and $S M 22 \alpha$-cre mouse lines have been previously described $(8,11,12,40)$. All animals were kept on a mixed C57BL/6:129/SvJ background. Genera- tion of lithium-treated embryos was performed as described (14). Pregnant females were given 2 intraperitoneal injections of $600 \mathrm{mM} \mathrm{LiCl}$ or $\mathrm{NaCl}$ (10 $\mu \mathrm{l} / \mathrm{g}$ body weight) between E8.5 and E10.5 of gestation. Embryos were collected either at E10.5 and lung buds isolated for Q-PCR analysis or at E14.5 and processed for immunohistochemical analysis.

Histology. Mouse tissue samples were harvested and fixed overnight in $4 \%$ paraformaldehyde, dehydrated in a series of ethanol washes, and embedded in paraffin. All results shown in the figures and table are representative of at least 4 samples of each genotype. Tissue sections were stained with the following antibodies: anti-SM22 $\alpha$ (Abcam; catalog AB10135), phosphohistone H3 (Cell Signaling Technology Inc.; catalog 9706L), anti-Tnc (Millipore; catalog AB1951), and anti-Pdgfr $\beta$ (Cell Signaling Technology Inc.; catalog 3169 ), using protocols previously described (4). $\beta$-Galactosidase histochemical staining was performed as previously described (8).

Cell culture. Pac1 cells were cultured in DMEM supplemented with $10 \%$ FBS and penicillin/streptomycin, as previously described (5). Cells were transfected with the indicated expression and reporter plasmids using FuGENE 6 (Roche Applied Science) and luciferase assays performed with the Dual-Glo assay system (Promega) according to the manufacturer's protocols. For knockdown experiments, cells were transfected with siRNA pools targeting $\beta$-catenin and Tnc (Thermo Fisher Scientific) using Lipofectamine 2000 (Invitrogen) according to the manufacturer's instructions. Reported values are the mean \pm SEM of 3 assays performed in duplicate.

Q-PCR and microarray analysis. Total RNA was isolated from cells and tissue with Trizol (Invitrogen) and reverse transcribed using Superscript II (Invitrogen) according to the manufacturer's protocols. Q-PCR was performed using the oligonucleotides listed in Supplemental Table 1 on an Applied Biosystems $7900 \mathrm{HT}$ system with SYBR Green reaction mixture (Applied Biosystems) according to the manufacturer's protocol. Reported values are the mean \pm SEM of 3 assays performed in duplicate. For microarray analysis, total RNA was isolated as previously described (4) from control and Wnt7b-expressing Pac-1 cells ( 2 samples each) and biotinylated cRNA probes generated for use as probes for Affymetrix rat 230A GeneChips (4). Two chips were hybridized for each condition. Array data were normalized using Microarray Suite 5.0 (MAS5; Affymetrix) and Significance Analysis of Microarrays (http://stat. stanford.edu/ tibs/SAM/). Changes in gene expression of 2.0-fold or greater, with $P$ values below 0.05 , were considered significant.

Lung explant cultures. Lung buds were explanted from E11.5 embryos using tungsten needles and cultured in BGjb media (Invitrogen) supplemented with $0.1 \mathrm{mg} / \mathrm{ml}$ ascorbic acid and penicillin / streptomycin for 48 hours, as previously described (4). At least 4 lung buds were used in each experiment. For antibody blocking experiments, explants were placed directly into media containing $25 \mu \mathrm{g} / \mathrm{ml}$ of either Tnc IgG (catalog AB1951; Millipore) or nonspecific control IgG. For Tnc protein treatment, explants were placed directly in either control media or media containing $10 \mu \mathrm{g} / \mathrm{ml}$ purified human Tnc protein (GeneWay Biotech). All explant experiments were repeated at least 3 times.

ChIP assays. Lung buds were isolated from wild-type embryos at E12.5, minced, fixed with $1 \%$ formaldehyde, and the chromatin sheared to an average length of 500-600 bp with a Branson Model 450 sonicator. Immunoprecipitation was performed with a commercially available kit (Upstate Biotechnology) and an antibody recognizing $\beta$-catenin (BD Biosciences; catalog 610154). After reverse cross-linking, precipitated chromatin was subjected to PCR and Q-PCR using the primers listed in Supplemental Table 1.

A. fumigatus-induced allergic asthma model. The method of sensitization and challenge with A. fumigatus has been previously described (24). Briefly, female $\mathrm{BALB} / \mathrm{c}$ mice were injected intraperitoneally with $20 \mu \mathrm{g}$ A. fumigatus combined with $2 \mathrm{mg}$ alum (Imject Alum; Pierce Biotechnology) in $100 \mu \mathrm{l}$ PBS on days 1 and 14 , followed by a single intranasal challenge on day 27 with $25 \mu \mathrm{g}$ of A. fumigatus extract (Hollister-Stier) in PBS (12.5 mg in $21 \%$ glycerol, PBS). 
Human lung tissue preparation. Lung sections from human pulmonary hypertension subjects were obtained through the Cardiovascular Medical Research Education Network and were processed by in accordance with institutional and NIH guidelines. Adult normal lung sections were obtained from ProSci. All protocols were reviewed and approved by the University of Alabama Institutional Review Board.

Statistics. A 2-tailed Student's $t$ test was used in these analyses. $P$ values less than 0.05 were considered statistically significant.

\section{Acknowledgments}

These studies were supported by funding from the NIH (HL075215 and HL087825 to E.E. Morrisey, HL087528 to E.D. Cohen, and HL079196 to P.L. Jones) and a Postdoctoral Fellowship grant from the Great Rivers Affiliate of the American Heart Association to E.D. Cohen. E.E. Morrisey is an Established Investigator of the American Heart Association.

Received for publication November 17, 2008, and accepted in revised form June 23, 2009.

Address correspondence to: Edward E. Morrisey, University of Pennsylvania, 956 BRB II/III, 421 Curie Blvd., Philadelphia, Pennsylvania 19104, USA. Phone: (215) 573-3010; Fax: (215) 573-2094; E-mail: emorrise@mail.med.upenn.edu. Or to: Ethan David Cohen, University of Pennsylvania, 938 BRB II/III, 421 Curie Blvd., Philadelphia, Pennsylvania 19104, USA. Phone: (215) 573-3010; Fax: (215) 573-2094; E-mail: cohene@mail.med. upenn.edu.
1. Cardoso, W.V., and Lu, J. 2006. Regulation of early lung morphogenesis: questions, facts and controversies. Development. 133:1611-1624.

2. Lane, K.B., et al. 2000. Heterozygous germline mutations in BMPR2, encoding a TGF-beta receptor, cause familial primary pulmonary hypertension. The International PPH Consortium. Nat. Genet. 26:81-84.

3. Deng, Z., et al. 2000. Familial primary pulmonary hypertension (gene PPH1) is caused by mutations in the bone morphogenetic protein receptor-II gene. Am. J. Hum. Genet. 67:737-744.

4. Zhang, Y., et al. 2008. A Gata6-Wnt pathway required for epithelial stem cell development and airway regeneration. Nat. Genet. 40:862-870.

5. Wang, Z., Shu, W., Lu, M.M., and Morrisey, E.E. 2005. Wnt7b activates canonical signaling in epithelial and vascular smooth muscle cells through interactions with Fzd1, Fzd10, and LRP5. Mol. Cell. Biol. 25:5022-5030.

6. Shu, W., et al. 2005. Wnt/beta-catenin signaling acts upstream of $\mathrm{N}$-myc, BMP4, and FGF signaling to regulate proximal-distal patterning in the lung. Dev. Biol. 283:226-239.

7. Mucenski, M.L., et al. 2003. beta-Catenin is required for specification of proximal/distal cell fate during lung morphogenesis. J. Biol. Chem. 278:40231-40238.

8. Shu, W., Jiang, Y.Q., Lu, M.M., and Morrisey, E.E. 2002. Wnt7b regulates mesenchymal proliferation and vascular development in the lung. Development. 129:4831-4842.

9. Weidenfeld, J., Shu, W., Zhang, L., Millar, S.E., and Morrisey, E.E. 2002. The WNT7b promoter is regulated by TTF-1, GATA6, and Foxa2 in lung epithelium. J. Biol. Chem. 277:21061-21070.

10. Jho, E.H., et al. 2002. Wnt/beta-catenin/Tcf signaling induces the transcription of Axin2, a negative regulator of the signaling pathway. Mol. Cell. Biol. 22:1172-1183.

11. Brault, V., et al. 2001. Inactivation of the betacatenin gene by Wnt1-Cre-mediated deletion results in dramatic brain malformation and failure of craniofacial development. Development. 128:1253-1264.

12. Lepore, J.J., et al. 2005. High-efficiency somatic mutagenesis in smooth muscle cells and cardiac myocytes in SM22alpha-Cre transgenic mice. Genesis. 41:179-184.

13. Hellstrom, M., Kalen, M., Lindahl, P., Abramsson, A., and Betsholtz, C. 1999. Role of PDGF-B and PDGFR-beta in recruitment of vascular smooth muscle cells and pericytes during embryonic blood vessel formation in the mouse. Development. 126:3047-3055.

14. Cohen, E.D., et al. 2007. Wnt/beta-catenin signaling promotes expansion of Isl-1-positive cardiac progenitor cells through regulation of FGF signaling. J. Clin. Invest. 117:1794-1804.

15. Hedgepeth, C.M., et al. 1997. Activation of the Wnt signaling pathway: a molecular mechanism for lithium action. Dev. Biol. 185:82-91.

16. Klein, P.S., and Melton, D.A. 1996. A molecular mechanism for the effect of lithium on development. Proc. Natl. Acad. Sci. U. S. A. 93:8455-8459.

17. Rajagopal, J., et al. 2008. Wnt7b stimulates embryonic lung growth by coordinately increasing the replication of epithelium and mesenchyme. Development. 135:1625-1634.

18. Ballagi, A.E., Ishizaki, A., Nehlin, J.O., and Funa, K. 1995. Isolation and characterization of the mouse PDGF beta-receptor promoter. Biochem. Biophys. Res. Commun. 210:165-173.

19. Jones, F.S., Meech, R., Edelman, D.B., Oakey, R.J., and Jones, P.L. 2001. Prx1 controls vascular smooth muscle cell proliferation and tenascin-C expression and is upregulated with Prx2 in pulmonary vascular disease. Circ. Res. 89:131-138.

20. Jones, P.L., Cowan, K.N., and Rabinovitch, M. 1997. Tenascin-C, proliferation and subendothelial fibronectin in progressive pulmonary vascular disease. Am. J. Pathol. 150:1349-1360.

21. Jones, P.L., and Rabinovitch, M. 1996. Tenascin$\mathrm{C}$ is induced with progressive pulmonary vascular disease in rats and is functionally related to increased smooth muscle cell proliferation. Circ. Res. 79:1131-1142.

22. Jones, P.L., Crack, J., and Rabinovitch, M. 1997. Regulation of tenascin-C, a vascular smooth muscle cell survival factor that interacts with the alpha $v$ beta 3 integrin to promote epidermal growth factor receptor phosphorylation and growth. J. Cell Biol. 139:279-293.

23. Ihida-Stansbury, K., et al. 2006. Tenascin-C is induced by mutated BMP type II receptors in familial forms of pulmonary arterial hypertension. Am.J. Physiol. Lung Cell Mol. Physiol. 291:L694-L702.

24. Haczku, A., et al. 2001. Aspergillus fumigatusinduced allergic airway inflammation alters surfactant homeostasis and lung function in BALB/c mice. Am. J. Respir. Cell Mol. Biol. 25:45-50.

25. Humbert, M., et al. 2004. Cellular and molecular pathobiology of pulmonary arterial hypertension. J. Am. Coll. Cardiol. 43:13S-24S.

26. Balasubramaniam, V., et al. 2003. Role of plateletderived growth factor in vascular remodeling dur- ing pulmonary hypertension in the ovine fetus. $A m$. J. Physiol. Lung Cell Mol. Physiol. 284:L826-L833.

27. Schermuly, R.T., et al. 2005. Reversal of experimental pulmonary hypertension by PDGF inhibition. J. Clin. Invest. 115:2811-2821.

28. Kaarteenaho-Wiik, R., et al. 2002. Tenascin-C is highly expressed in respiratory distress syndrome and bronchopulmonary dysplasia. J. Histochem. Cytochem. 50:423-431.

29. Konigshoff, M., et al. 2008. Functional Wnt signaling is increased in idiopathic pulmonary fibrosis. PLOS ONE. 3:e2142.

30. Nakahara, H., et al. 2006. Deficiency of tenascin C attenuates allergen-induced bronchial asthma in the mouse. Eur. J. Immunol. 36:3334-3345.

31. Lewis, C.C., et al. 2003. Increased expression of platelet-derived growth factor receptor-beta in airway fibroblasts of severe asthmatics. Chest. 123:428S-429S.

32. Matsuda, A., et al. 2005. Coding SNP in tenascinC Fn-III-D domain associates with adult asthma. Hum. Mol. Genet. 14:2779-2786.

33. Mucenski, M.L., et al. 2005. Beta-catenin regulates differentiation of respiratory epithelial cells in vivo. Am. J. Physiol. Lung Cell Mol. Physiol. 289:L971-L979.

34. Wu, L.S., Tan, C.Y., Wang, L.M., Lin, C.G., and Wang, J.Y. 2006. Variant in promoter region of platelet-derived growth factor receptor-alpha (PDGFRalpha) gene is associated with the severity and allergic status of childhood asthma. Int. Arch. Allergy Immunol. 141:37-46.

35. De Langhe, S.P., et al. 2005. Dickkopf-1 (DKK1) reveals that fibronectin is a major target of Wnt signaling in branching morphogenesis of the mouse embryonic lung. Dev. Biol. 277:316-331.

36. Parr, B.A., Avery, E.J., Cygan, J.A., and McMahon, A.P. 1998. The classical mouse mutant postaxial hemimelia results from a mutation in the Wnt 7 a gene. Dev. Biol. 202:228-234.

37. Li, C., Xiao, J., Hormi, K., Borok, Z., and Minoo, P. 2002. Wnt5a participates in distal lung morphogenesis. Dev. Biol. 248:68-81.

38. Li, C., et al. 2005. Wnt5a regulates Shh and Fgf10 signaling during lung development. Dev. Biol. 287:86-97.

39. Que, J., et al. 2008. Mesothelium contributes to vascular smooth muscle and mesenchyme during lung development. Proc. Natl. Acad. Sci. U. S. A. 105:16626-16630.

40. Maretto, S., et al. 2003. Mapping Wnt/betacatenin signaling during mouse development and in colorectal tumors. Proc. Natl. Acad. Sci. U. S. A. 100:3299-3304. 